\title{
Guillain-Barré Syndrome after Lung Transplantation in the Immediate Postoperative Period: Case Report
}

\author{
Byung Mo Gu, M.D. ${ }^{1}$, Ho Hyun Ko, M.D. ', Hong Kyu Lee, M.D. ', Yong Joon Ra, M.D. ', Hee Sung Lee, M.D., Ph.D. ${ }^{\text {, }}$ \\ Hyoung Soo Kim, M.D., Ph.D. ${ }^{1}$
}

'Department of Thoracic and Cardiovascular Surgery, Hallym University Sacred Heart Hospital, Anyang; ${ }^{2}$ Department of Thoracic and Cardiovascular Surgery, Hallym University Dongtan Sacred Heart Hospital, Hwaseong, Korea

\author{
ARTICLE INFO \\ Received June 11, 2020 \\ Revised September 23, 2020 \\ Accepted October 4, 2020 \\ Corresponding author \\ Hyoung Soo Kim \\ Tel $82-31-380-3815$ \\ Fax 82-31-380-4118 \\ E-mail cskhs99@hallym.or.kr \\ ORCID \\ https://orcid.org/0000-0001-6023-0818
}

\begin{abstract}
A 58-year-old man, incapable of maintaining oxygen saturation with mechanical ventilation, was admitted to our hospital for veno-venous extracorporeal membrane oxygenation (ECMO) treatment. He was diagnosed with acute respiratory distress syndrome (ARDS) due to influenza A pneumonia. His condition stabilized with antibiotics and steroid administration, but weaning from ECMO failed due to post-infectious pulmonary sequelae. On day 84 after admission, he underwent bilateral lung transplantation. In the postoperative phase, he did not regain consciousness even after discontinuation of sedatives for 3 days. However, spontaneous pupillary reflex and eye movements were preserved, while communication and upper and lower limb movements were affected. The nerve conduction study was diagnostic of Guillain-Barré syndrome. He was managed with intravenous immunoglobulins and plasmapheresis. Mild recovery of the facial muscles was seen, but he died 24 days post-surgery due to progressive ARDS and sepsis.
\end{abstract}

Keywords: Guillain-Barre syndrome, Lung transplantation, Extracorporeal circulation, Complication, Acute respiratory distress syndrome, Case report

\section{Case report}

A 58-year-old man was admitted to a local hospital because of fever, dyspnea, and cough with expectoration. His chest radiograph showed bilateral consolidation of the lungs, which was suggestive of pneumonia; therefore, he was started on antibiotic treatment. Since his oxygen saturation continued to decrease, he was intubated and kept on a ventilator. After a week of hospitalization, oxygen saturation still could not be maintained with mechanical ventilation. He was then transferred to Hallym University Sacred Heart Hospital for veno-venous extracorporeal membrane oxygenation (ECMO) treatment. We performed a respiratory viral panel test, which led to the diagnosis of acute respiratory distress syndrome (ARDS) due to influenza A pneumonia. After a few weeks of treatment with awake veno-venous ECMO, the levels of inflammatory markers normalized, and his general condition improved. However, he could not be weaned from ECMO due to post-infectious pulmonary sequelae. A chest computed tomography scan on the 67th day after admission showed extensive ground glass opacities and diffuse bronchiectasis in both lungs (Fig. 1). Bilateral lung transplantation was performed on the 84th day of hospital stay. In the operation, an arterial cannula was inserted into the ascending aorta and 2 cannulas of veno-venous ECMO were connected and used as a venous cannula to convert the setup to central veno-arterial ECMO. The ischemic time of the right donor lung was 3 hours and that of the left donor lung was 4 hours and 56 minutes. After anastomosis finished, weaning from ECMO was attempted, but the patient's blood pressure was not maintained even with sufficient inotropes and fluids due to decreased heart function. Eventually, a single arterial cannula was inserted into the femoral artery to change central veno-arterial ECMO into peripheral ECMO, and the operation was completed. Induction therapy with methylprednisolone $(500 \mathrm{mg})$ was administered intravenously. An immunosuppressive regimen consisting of tacrolimus (target therapeutic range, $5-14 \mu \mathrm{g} / \mathrm{mL}$ ) and mycophenolate mofetil (1,000 mg/day) was started after transplantation. 


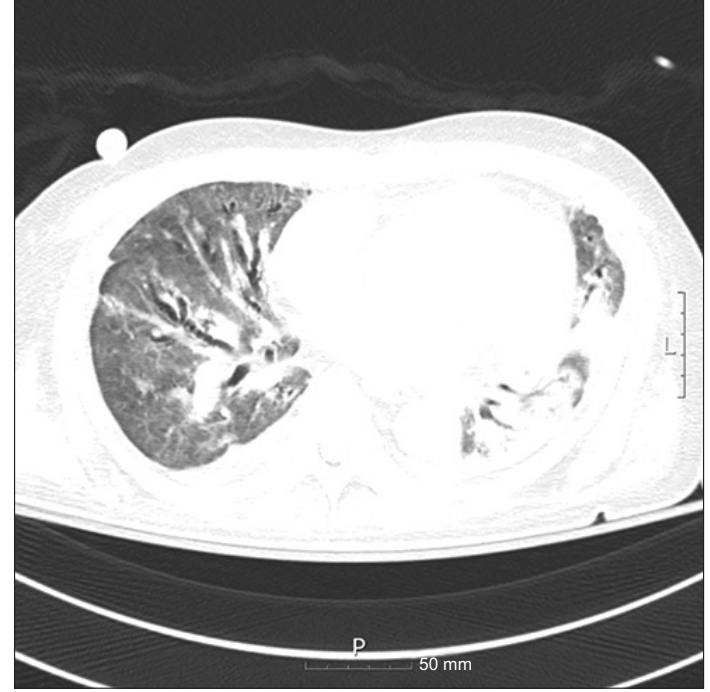

Fig. 1. Chest computed tomography image showing extensive ground glass opacities and diffuse bronchiectasis in the bilateral lungs.

Due to bleeding owing to coagulopathy after surgery, he was re-operated on days 1 and 2 after transplantation. Thereafter, sedatives were stopped, and we waited for the patient to regain consciousness. However, even 3 days after the second operation for bleeding control, he was unresponsive and showed no movement of the upper and lower extremities except for spontaneous pupil and eye movement. Deep tendon reflexes of the patient's limbs were absent, and a nerve conduction study was performed to investigate the cause. The results were suggestive of sensory-motor polyneuropathy (motor-dominant demyelinating neuropathy) (Table 1). Cerebrospinal fluid testing was not performed due to the patient's poor general condition and bleeding tendency. No electrolyte abnormalities or other causes were found. Guillain-Barré syndrome (GBS) was diagnosed based on its typical clinical manifestations. We started intravenous immunoglobulin therapy (IVIG) and plasmapheresis, which are the conventional treatments for GBS. Bronchoscopic toileting was performed regularly for effective lung care. As the treatment progressed, mild recovery of facial muscles was seen, but the patient died 24 days after surgery due to progression of ARDS and sepsis.

The patient's spouse provided written informed consent for the publication of his clinical details and images.

\section{Discussion}

GBS is a rare disease characterized by acute areflexic paralysis due to damage to the peripheral nervous system through an impaired immune response. The incidence of GBS is reported as 0.89 to 1.89 cases per 100,000 personyears, with a male-to-female ratio of 1.78 [1]. Although the immuno-pathogenesis of this disease has not been clearly identified, its basic mechanism is an inappropriate autoimmune response that damages the myelin sheath of neurons. The treatments available include IVIG and plasmapheresis. However, GBS has a poor prognosis, since $20 \%$ of the patients have persistent disability and approximately $5 \%$ die from medical complications [2]. Infections, vaccination, and surgery are known to increase its incidence.

GBS is difficult to diagnose because there are no biomarkers with high specificity and sensitivity. Therefore, the diagnosis of GBS is based on clinical manifestations and ancillary laboratory investigations and the commonly used criteria presented by the National Institute of Neurological Disorders and Stroke, which consist of features required for diagnosis, features strongly supportive of diagnosis, and features that rule out diagnosis [3]. In this case, the patient showed progressive motor weakness and areflexia, which are features required for diagnosis. Several strongly supportive features were also observed: symmetry of symptoms, cranial nerve involvement, autonomic dysfunction, and electrodiagnostic features of sensory-motor neuropathy.

Since GBS involves an autoimmune mechanism, it was thought that transplant patients undergoing immunosuppressive management would be protected from GBS. However, there have been many reports of GBS being associated with transplantation. In case of bone marrow transplantation, the incidence of GBS is reported to be $0.3 \%-0.7 \%$, which is much higher than that in the general population [4]. A study reported by El-Sabrout et al. [5] regarding GBS cases occurring after solid organ transplantation indicated that cytomegalovirus infection plays an important role in the development of GBS in transplant patients. However, several case reports have also been published wherein GBS was reported to occur after transplantation without any history of infection [6,7]. These reports suggested that tacrolimus, an immunosuppressive drug classified as a calcineurin inhibitor and used after solid organ transplantation, may cause GBS. Neurotoxicity related to calcineurin inhibitors, such as tacrolimus and cyclosporine, is well described in the literature; furthermore, it has been claimed that neurotoxicity associated with these drugs may be reversed by reducing the dosage [8].

In our patient, a history of influenza A infection, major surgery, and administration of tacrolimus were the risk factors for GBS. Unlike other cases in which GBS occurred 
Table 1. Motor nerve conduction and sensory nerve conduction

\begin{tabular}{|c|c|c|c|c|c|}
\hline Nerve and site & Latency (msec) & Amplitude (mV) & Segment & Distance $(\mathrm{mm})$ & $\mathrm{CV}(\mathrm{m} / \mathrm{sec})$ \\
\hline \multicolumn{6}{|l|}{ Motor nerve conduction } \\
\hline \multicolumn{6}{|l|}{ Tibial (left) } \\
\hline Ankle & 4.7 & 2.5 & $\mathrm{AH}$-ankle & & \\
\hline PF & 15.2 & 1.5 & Ankle-PF & 350 & 33 \\
\hline \multicolumn{6}{|l|}{ Tibial (right) } \\
\hline Ankle & 4.3 & 2.6 & $\mathrm{AH}$-ankle & & \\
\hline PF & 13.9 & 1.4 & Ankle-PF & 380 & 40 \\
\hline \multicolumn{6}{|l|}{ Median (left) } \\
\hline Wrist & 4.2 & 2.3 & APB-wrist & & \\
\hline Elbow & 8.9 & 2.3 & Wrist-elbow & 225 & 48 \\
\hline Axilla & 11.2 & 2.3 & Elbow-axilla & 100 & 43 \\
\hline \multicolumn{6}{|l|}{ Ulnar (left) } \\
\hline Wrist & 2.9 & 1.5 & ADQ-wrist & & \\
\hline $\mathrm{BE}$ & 7.5 & 1.3 & Wrist-BE & 240 & 52 \\
\hline $\mathrm{AE}$ & 9.2 & 1.2 & $\mathrm{BE}-\mathrm{AE}$ & 80 & 47 \\
\hline Axilla & 10.5 & 1.1 & AE-axilla & 80 & 62 \\
\hline \multicolumn{6}{|l|}{ Median (right) } \\
\hline Wrist & 4.5 & 3.3 & APB-wrist & & \\
\hline Elbow & 9.8 & 3.2 & Wrist-elbow & 240 & 45 \\
\hline Axilla & 11.8 & 3.2 & Elbow-axilla & 100 & 50 \\
\hline \multicolumn{6}{|l|}{ Ulnar (right) } \\
\hline Wrist & 3.3 & 2.7 & ADQ-wrist & & \\
\hline $\mathrm{BE}$ & 8.7 & 1.5 & Wrist-BE & 235 & 44 \\
\hline $\mathrm{AE}$ & 10.4 & 1.5 & $\mathrm{BE}-\mathrm{AE}$ & 80 & 47 \\
\hline Axilla & 11.6 & 1.2 & AE-axilla & 80 & 67 \\
\hline \multicolumn{6}{|c|}{ Sensory nerve conduction } \\
\hline \multicolumn{6}{|l|}{ Median (left) } \\
\hline Digit III & 2.8 & 30 & R1-digit III & 125 & 45 \\
\hline Mid-palm & 2.3 & 44 & R1-mid palm & 85 & 37 \\
\hline Wrist & 4.9 & 9 & R1-wrist & 230 & 47 \\
\hline Elbow & 2.6 & 9 & R1-elbow & 110 & 38 \\
\hline \multicolumn{6}{|l|}{ Ulnar (left) } \\
\hline Digit V & 3.4 & 8 & R2-digit V & 125 & 37 \\
\hline Wrist & 5.2 & 7 & R2-wrist & 240 & 46 \\
\hline Elbow & 2.3 & 12 & R2-elbow & 100 & 43 \\
\hline \multicolumn{6}{|l|}{ Median (right) } \\
\hline Digit III & 3.5 & 27 & R1-digit III & 130 & 37 \\
\hline Mid palm & 3.0 & 42 & R1-mid palm & 90 & 30 \\
\hline Wrist & 5.1 & 13 & R1-wrist & 230 & 45 \\
\hline Elbow & 2.2 & 56 & R1-elbow & 100 & 45 \\
\hline \multicolumn{6}{|l|}{ Ulnar (right) } \\
\hline Digit V & 3.5 & 11 & R2-digit V & 120 & 34 \\
\hline Wrist & 5.3 & 13 & R2-wrist & 240 & 45 \\
\hline Elbow & 2.0 & 6 & R2-elbow & 100 & 50 \\
\hline
\end{tabular}

The nerve conduction study showed delayed distal latency, reduction of amplitude, and slowed nerve conduction velocities in both motor and sensory nerves. This sensory-motor polyneuropathy (motor-dominant demyelinating neuropathy) was suggestive of Guillain-Barré syndrome.

$\mathrm{AH}$, abductor hallucis; PF, popliteal fossa; $\mathrm{APB}$, abductor pollicis brevis; $\mathrm{ADQ}$, abductor digiti quinti; $\mathrm{BE}$, below elbow; $\mathrm{AE}$, above elbow; R1/2, recording site.

weeks to months after transplantation [4-6], this patient developed GBS in the immediate postoperative period. To the best of our knowledge, there have been no reports of the development of GBS immediately after transplantation.
It was difficult for us to decide whether the patient's unconsciousness was due to sedation by an anesthetic drug or the symptoms of the disease.

The patient received veno-arterial ECMO owing to car- 
diogenic shock that occurred during surgery. The patient's echocardiography findings were normal before transplantation and there were no other causes of acute heart failure. However, there have been reports of stress cardiomyopathy as a rare complication of GBS [9]. Therefore, the deterioration of the cardiac function in this patient is likely to have been associated with the development of GBS.

Although we considered discontinuation of tacrolimus, we did not stop it because of concerns regarding acute rejection. Given the patient's condition, discontinuation of tacrolimus to restore spontaneous movement and respiratory function rapidly might have been more helpful. Since spontaneous breathing and expectoration of sputum are important in lung transplantation, we performed bronchoscopic toileting to prevent pneumonia. However, in immunosuppressed patients who are unable to move and cannot breathe spontaneously, the progression of sepsis cannot be prevented due to the exacerbation of pneumonia.

Patients undergoing lung transplantation have many risk factors for the development of GBS. They are usually vaccinated for pneumonia before transplantation and continue to take tacrolimus after surgery. In addition, as major surgery, lung transplantation itself is a tremendous risk factor. If GBS develops in a stable state several weeks to months after surgery, one can focus on its treatment and choose to discontinue tacrolimus. However, if it occurs immediately after surgery as in the present case, there are many limitations regarding treatment and diagnosis.

From this case, we can learn that it is always helpful to keep in mind that GBS may occur immediately after transplantation, and it should also be noted that stress cardiomyopathy may develop as a complication of GBS.

\section{Conflict of interest}

No potential conflict of interest relevant to this article was reported.

\section{ORCID}

Byung Mo Gu: https://orcid.org/0000-0001-9632-6803

Ho Hyun Ko: https://orcid.org/0000-0002-2612-5026

Hong Kyu Lee: https://orcid.org/0000-0002-9087-7783

Yong Joon Ra: https://orcid.org/0000-0003-2153-504X

Hee Sung Lee: https://orcid.org/0000-0002-2615-8346

Hyoung Soo Kim: https://orcid.org/0000-0001-6023-0818

\section{References}

1. Sejvar JJ, Baughman AL, Wise M, Morgan OW. Population incidence of Guillain-Barre syndrome: a systematic review and meta-analysis. Neuroepidemiology 2011;36:123-33.

2. Hughes RA, Swan AV, Raphael JC, Annane D, van Koningsveld R, van Doorn PA. Immunotherapy for Guillain-Barre syndrome: a systematic review. Brain 2007;130(Pt 9):2245-57.

3. Asbury AK, Cornblath DR. Assessment of current diagnostic criteria for Guillain-Barre syndrome. Ann Neurol 1990;27 Suppl:S21-4.

4. Wen PY, Alyea EP, Simon D, Herbst RS, Soiffer RJ, Antin JH. Guillain-Barre syndrome following allogeneic bone marrow transplantation. Neurology 1997;49:1711-4.

5. El-Sabrout RA, Radovancevic B, Ankoma-Sey V, van Buren CT. Guillain-Barre syndrome after solid organ transplantation. Transplantation 2001;71:1311-6.

6. Sharma NS, Wille KM, Hoopes CW, Diaz-Guzman E. Acute demyelinating polyneuropathy after lung transplantation: Guillain-Barre syndrome or tacrolimus toxicity? Case Rep Transplant 2014;2014: 685010.

7. Meena P, Bhargava V, Rana DS, et al. Tacrolimus-associated Guillain-Barre syndrome. Am J Ther 2020 Feb 20 [Epub]. https://doi. org/10.1097/MJT.0000000000001096.

8. Bechstein WO. Neurotoxicity of calcineurin inhibitors: impact and clinical management. Transpl Int 2000;13:313-26.

9. Boon M, Dennesen PJ, Veldkamp RF. A rare stress cardiomyopathy in a patient with Guillain-Barre syndrome. Neth J Med 2016;74:868. 